University of Nebraska - Lincoln

DigitalCommons@University of Nebraska - Lincoln

$11-1-2002$

\title{
Confirmatory factor analysis of the Behavior Rating Inventory of Executive Function (BRIEF) in a clinical sample
}

\author{
G. G. Gioia \\ P. K. Isquith \\ Dartmouth Medical School, Isquith@Dartmouth.edu \\ P. D. Retzlaff \\ K. A. Espy \\ University of Nebraska-Lincoln, kespy2@unl.edu
}

Follow this and additional works at: https://digitalcommons.unl.edu/dcnlfacpub

Part of the Neurosciences Commons

Gioia, G. G.; Isquith, P. K.; Retzlaff, P. D.; and Espy, K. A., "Confirmatory factor analysis of the Behavior Rating Inventory of Executive Function (BRIEF) in a clinical sample" (2002). Developmental Cognitive Neuroscience Laboratory - Faculty and Staff Publications. 15.

https://digitalcommons.unl.edu/dcnlfacpub/15

This Article is brought to you for free and open access by the Developmental Cognitive Neuroscience Laboratory at DigitalCommons@University of Nebraska - Lincoln. It has been accepted for inclusion in Developmental Cognitive Neuroscience Laboratory - Faculty and Staff Publications by an authorized administrator of DigitalCommons@University of Nebraska - Lincoln. 


\title{
Confirmatory Factor Analysis of the Behavior Rating Inventory of Executive Function (BRIEF) in a Clinical Sample
}

\author{
Gerard A. Gioia', Peter K. Isquith ${ }^{2}$, Paul D. Retzlaff ${ }^{3}$, and Kimberly A. Espy ${ }^{4}$ \\ 'Children's National Medical Center, Washington, DC, USA, ${ }^{2}$ Dartmouth Medical School, Hanover, NH, USA, \\ ${ }^{3}$ University of Northern Colorado, Greeley, CO, USA, and ${ }^{4}$ Southern Illinois University School of Medicine, \\ Carbondale, IL, USA
}

\begin{abstract}
Evidence for the validity of the Behavior Rating Inventory of Executive Function (BRIEF; Gioia, Isquith, Guy, \& Kenworthy, 2000) based on internal structure was examined in a sample of children with mixed clinical diagnoses via maximum likelihood confirmatory factor analysis. Four alternative factor models of children's executive function, based on current theories that posit a unidimensional versus fractionated model (Rabbitt, 1997; Shallice \& Burgess, 1991), using the revised 9-scale BRIEF configuration that separates two components of the Monitor scale, were examined for model fit. A 3-factor structure best modeled the data when compared directly with 1-,2-, and 4-factor models. The 3-factor model was defined by a Behavior Regulation factor consisting of the BRIEF Inhibit and Self-Monitor scales, an Emotional Regulation factor consisting of the Emotional Control and Shift scales, and a Metacognition factor composed of the Working Memory, Initiate, Plan/Organize, Organization of Materials, and Task-Monitor scales. The findings support a fractionated, multi-component view of executive function as measured by the BRIEF.
\end{abstract}

The Behavior Rating Inventory of Executive Function (BRIEF; Gioia et al., 2000) is a parentand teacher-completed rating scale, developed to assess the everyday behavioral manifestations of children's executive control functions. As published, its eight scales comprise a Behavioral Regulation Index and a Metacognition Index, which in turn comprise a Global Executive Composite. The scale-to-index configuration is based on the theoretical assumption that these regulatory functions are, to some extent, separable in a clinically meaningful way, yet related within an overarching executive system. These theoretical assumptions, and the BRIEF scales, require further investigation to explore the validity of their underlying structure. The present paper examines alternative factor models of the BRIEF via confirmatory factor analysis to further specify the structure of the clinical instrument. The analysis also sheds light on the nature of the construct of executive function.

Debate continues regarding acceptance of executive functions as a unitary versus multidimensional construct (Goldman-Rakic, 1987; Kelly, 2000; Welsh, Pennington, \& Grossier, 1991). Definitions of the executive functions typically refer to a collection of related, though possibly distinct, abilities that direct and control

The authors maintain financial interest in the BRIEF as developers of that instrument. No financial support was provided for any phase of this research.

Address correspondence to: Gerard A. Gioia, Pediatric Neuropsychology Program, Children's National Medical Center, 4900 Massachusetts Avenue, Suite 320, Washington, DC, 20016, USA. E-mail: ggioia@cnmc.org Accepted for publication: October 6, 2002. 
goal-oriented cognitive, behavioral, and emotional functioning. Discussions of specific executive functions include anticipating, planning and organizing, initiating action plans, inhibiting distractions and interference, monitoring the process, and shifting flexibly to new actions when necessary, all the while holding these processes and a goal state in active working memory. Definitions differentiate more "basic" cognitive functions (e.g., language, visual-spatial, memory processes) from the "executive" or directive, cognitive control functions (Denckla, 1996). Although specific subdomains of the executive functions can be debated, most would agree that the term is an umbrella construct for a collection of interrelated functions. Yet the question still remains: Is the construct of executive function a unitary entity or are the subdomains separable?

Rabbit (1997) posits that, although various executive functions can be observed behaviorally in different tasks and/or situations, the underlying processes may not be distinct or independent. The problem-solving process argues for interrelations of executive components to achieve efficient multi-step performance. Certain executive functions (e.g., inhibition) may be more primary, playing an underlying "enabling" role (Barkley, 1997, 2000; Burgess, 1997) that provides for sustained strategic problem-solving. Burgess (1997) discusses the concept of reciprocal causation with the executive functions, whereby inhibition is necessary to facilitate higher level "meta" cognitive strategy use, lessening the cognitive load of a task, which in turn reduces the need for inhibition. Barkley (1997) posits a model of executive function within the disorder of ADHD that views inhibition as the fundamental underlying factor that enables other functions including working memory (verbal and nonverbal), emotional regulation, and goal-directed analysis and synthesis in problem-solving (reconstitution). His theoretical model partitions the executive functions into separable, though integrated, components.

Both inhibitory and higher level metacognitive components are important executive controls in task performance but may be difficult to disentangle from a methodological and statistical point of view. In their study of patients with frontal lobe lesions, however, Shallice and Burgess (1991) report quantitative support for fractionation of the supervisory system into specific executive functions with novel task methodologies and particular attention to everyday behavior.

In addition to theoretical complexity, measurement of executive function is inherently challenging. Efforts to operationalize assessment models of executive function have largely focused on laboratory or clinical performance tests (Kelly, 2000; Levin et al., 1991; Welsh \& Pennington, 1988; Welsh et al., 1991), but these methods have inherent construct and measurement problems (Pennington \& Ozonoff, 1996; Rabbit, 1997). Clinical tests that purport to assess executive functions necessarily confound content and method with control processes. Shared method variance associated with test content (e.g., vocabulary skill in verbal fluency) may give the appearance of defining unique, separable executive control dimensions while not separating content related variance. Burgess (1997) suggests that most neuropsychological tests alone are inadequate in assessing the executive functions because they attempt to separate integrated functions into component parts. Furthermore, current performance-based tests tap individual cognitive components of executive function over a short time frame and not the integrated, multidimensional, relativistic, priority-based decision-making that is often demanded in real world situations (Goldberg \& Podell, 2000; Shallice \& Burgess, 1991). As a result, narrow-band, component tests may not be sufficient to capture more complex everyday executive problem-solving. Given that there is no "prototypical screening measure," assessment may require many hours of testing and consultation with people who know the patient well (Burgess, 1997). Thus, there is a role for ecologically valid methods of measuring executive function that assess the broader, molar aspects of complex, everyday problem-solving demands.

The BRIEF was developed to assess executive function from a more everyday, molar behavioral perspective. To some extent, the behavior rating scale methodology circumvents the cognitive "slave" system (e.g., language, visual/nonverbal systems), problematic with the use of performance tests, and offers a view of executive function in 
the everyday world rather than in isolation on clinic-based performance tests (Gioia \& Isquith, 2002 b; Silver, 2000). Exploratory factor analysis of the eight scales comprising the BRIEF consistently identified a 2-factor structure within parent ratings and teacher ratings for both normative and clinical groups: a 3-scale (Inhibit, Shift, and Emotional Control) Behavioral Regulation factor and a 5-scale (Initiate, Working Memory, Plan/Organize, Organization of Materials, and Monitor) Metacognition factor (Gioia et al., 2000). Recently, the item content of the Monitor scale was re-examined and hypothesized to reflect two distinct dimensions - monitoring of taskrelated activities and monitoring of personal behavioral activities (Gioia \& Isquith, 2002). The two monitoring dimensions - task-monitoring and self-monitoring - were found to be reasonably stable over time with test-retest reliabilities of .78 and .82 , respectively, and only moderately correlated with each other $(r=.47)$. Further, the two subcomponents of the Monitor scale associated differently with the Behavioral Regulation and Metacognition Indexes in exploratory factor analysis. The task-monitoring scale loaded strongly with the five Metacognition scales while the selfmonitoring scale loaded with the three Behavioral Regulation scales.

In the current investigation, competing models of the underlying structure of the BRIEF are examined explicitly using the revised 9-scale configuration that separates the two components of the Monitor scale via parent ratings of children with mixed clinical diagnoses. Confirmatory factor analysis (CFA) allows an objective test of the statistical fit against the observed data of alternative models of executive function that are specified a priori, which provides more systematic support of the validity of the BRIEF than is possible with exploratory methods.

Four competing theoretical models of executive function using the BRIEF in a clinical sample were examined for model fit and parsimony. We considered only models that (a) were clinically interpretable and (b) had at least two scales per factor. First, a baseline single-factor model was fit that considered executive functioning as a unitary construct. Therefore, in Model 1, all scales were constrained to load on one latent factor. Model 2 was defined as a 2-factor model with four scales (Inhibit, Shift, Emotional Control, and Self-Monitor) loading on the latent Behavioral Regulation factor and five scales (Initiate, Working Memory, Plan/Organize, Organization of Materials, and Task-Monitor) loading on the latent Metacognition factor. In Model 3, the Behavioral Regulation factor from Model 2 was parsed further, such that the Inhibit and SelfMonitor scales comprised the latent Behavioral Regulation factor, and the BRIEF Emotional Control and Shift scales loaded on the latent Emotional Regulation factor. In Model 3, the latent Metacognition factor again was composed of the Initiate, Working Memory, Plan/Organize, Organization of Materials, and Task-Monitor scales. Model 4 included a 4 -factor solution, retaining the parsed Behavioral and Emotional Regulation latent factors, and further fractionating the Metacognition factor into two latent factors. The latter two factors were labeled "Internal" Metacognition, composed of the BRIEF Initiate, Working Memory, and Plan/ Organize scales, and "External" Metacognition, composed of Organization of Materials and TaskMonitor scales.

\section{METHOD}

\section{Participants}

A sample of 374 children ( 272 boys, 112 girls) aged 5-18 years $(M=9.06$ years, $S D=2.73$ years $)$ were selected for the study. Diagnoses for the sample were mixed, including Attention-Deficit/Hyperactivity Disorder (ADHD), learning disabilities, Autism Spectrum Disorders (ASD), Tourette Syndrome, affective disorders, and seizure disorders. The sample was diverse in terms of parent education with a range of 6-24 years (maternal education $M=14.27, S D=2.78$; paternal education $M=13.96, S D=3.07)$. Participants were recruited from two sources: clinical referrals $(n=241)$ and parent support groups $(n=133)$.

\section{Measure}

Parents completed the Behavior Rating Inventory of Executive Function - Parent Form (BRIEF; Gioia et al. 2000). The BRIEF is composed of 86 items in which the parent responds whether their child exhibits problems with specific behaviors: Never, Sometimes, or Often, scored as 1, 2, or 3, respectively. 


\section{Design and Analysis}

The latent factor structure of the nine scales of the BRIEF was examined via maximum likelihood confirmatory factor analysis using the EQS program (Bentler \& Wu, 1995). Mean raw score ratings (range 1-3) for each of the nine BRIEF scales (Inhibit, Shift, Emotional Control, Initiate, Working Memory, Plan/ Organize, Organization of Materials, and Monitor subdivided into Task-Monitoring and Self-Monitoring) were entered as measured variables in the four a priori models. The confirmatory factor analyses were conducted on the covariance matrix, as all BRIEF scales share the same metric.

The four models were compared for their adequacy of fit using $\mathrm{Hu}$ and Bentler's (1998, 1999) recommended approach to fit criteria. First, the two-index presentation strategy was used, requiring a standardized root mean-square residual (SRMR) of $\leq .08$ and a comparative fit index (CFI) of $\geq .95$. Second, models were examined for values of the root mean squared error of approximation (RMSEA) of $\leq .06$ and a $x^{2} / d f$ ratio $\leq 5$. Finally, the largest standardized residual and average off-diagonal absolute standardized residual were examined, with lower values preferred. Because the models are nested, direct comparison of the differences in model fit was possible through examination of incremental change in $\chi^{2}$.

\section{RESULTS}

Table 1 provides the means and standard deviations for the BRIEF variables. The BRIEF descriptive statistics suggest that all scales were moderately elevated, consistent with that seen in previous samples of children with mixed clinical

Table 1. BRIEF Scale Means and Standard Deviations.

\begin{tabular}{lcc}
\hline BRIEF scale & Mean & SD \\
\hline Initiate & 2.05 & 0.47 \\
Working Memory & 2.40 & 0.45 \\
Plan/Organize & 2.21 & 0.51 \\
Organization of & 2.40 & 0.57 \\
$\quad$ Materials & 2.42 & 0.50 \\
Task-Monitor & 2.14 & 0.62 \\
Inhibit & 2.25 & 0.59 \\
Self-Monitor & 1.94 & 0.54 \\
Shift & 2.09 & 0.58 \\
Emotional Control & & \\
\hline
\end{tabular}

Note. Range of scores 1 (Never)-3 (Often). diagnoses (Gioia et al., 2000). The clinical sample exhibited means on the order of $2.0-2.4$ and standard deviations near 0.50 versus means of approximately 1.5 and standard deviations of 0.40 in the normative sample (Gioia et al., 2000). Correlations among the nine BRIEF scales are presented in Table 2. Generally, the patterns of correlations between scales that range between .3 and .7 suggest differential relationships,

Table 3 summarizes the fit indices for the four models. The baseline, single factor model fit the data poorly in comparison to all fit criteria. The incremental fit of the 2-factor model differed from the baseline, $\chi^{2}(1)=298, p<.0001$. The fit of the 2 -factor model, however, was poor relative to the observed data, with a CFI less than the .95 criterion, although the SRMR was .05, less than the criterion of $\leq .08$. The incremental difference in fit between Models 3 and 2 also differed, $\chi^{2}(2)=42$, $p<.001$. The 3-factor model, including latent Behavioral Regulation, Emotional Regulation, and Metacognition factors, fit the observed data well. The CFI was .95, with an SRMR of .04. The RMSEA of this model was larger (.11) than the desired value, $\leq .06$. The highest standardized residual was .12 , between BRIEF Plan/Organize and Inhibit Scales. The incremental fit of the 4-factor model that further parsed the latent Metacognition into "Internal" versus "External" referents did not differ from the 3-factor model, $\chi^{2}(3)=6, p>05$. The CFI and SRMR for Model 4 were comparable to that of Model 3 (.95 and .04 respectively), but the RMSEA was slightly larger at .12. Given the lack of difference in incremental fit and comparable fit indices, Model 3 was preferred as it offers a simpler, more parsimonious model of the observed data.

The latent factor solution for Model 3 is depicted in Figure 1. As shown, all factor loadings exceeded, 65 , supporting the hypothesized relations between the BRIEF scales and the three latent executive factors. Not surprisingly, the Behavioral Regulation and Emotional Regulation factors were correlated highly $(r=84)$, with lower correlations between these latent factors and the Metacognition factor $(r=.63$ and $r=64$, respectively). The magnitude of the correlations between the latent factors indicated a noteworthy degree of shared variance between 
Table 2. BRIEF Scale Intercorrelations.

\begin{tabular}{|c|c|c|c|c|c|c|c|c|c|}
\hline BRIEF scale & 1 & 2 & 3 & 4 & 5 & 6 & 7 & 8 & 9 \\
\hline 1. Initiate & - & & & & & & & & \\
\hline 2. Working Memory & .61 & - & & & & & & & \\
\hline 3. Plan/Organize & .72 & .71 & - & & & & & & \\
\hline $\begin{array}{l}\text { 4. Organization } \\
\text { of Materials }\end{array}$ & .50 & .56 & .57 & - & & & & & \\
\hline 5. Task-Monitor & .48 & .54 & .63 & .45 & - & & & & \\
\hline 6. Inhibit & .36 & .51 & .36 & .45 & .33 & - & & & \\
\hline 7. Self-Monitor & .49 & .54 & .47 & .39 & .37 & .73 & - & & \\
\hline 8. Shift & .49 & .47 & .50 & .30 & .38 & .54 & .57 & - & \\
\hline 9. Emotional Control & .42 & .46 & .38 & .36 & .30 & .66 & .59 & .68 & - \\
\hline
\end{tabular}

Note. $N=374$; All correlations significant at $p<.01$.

Table 3. Summary of Fit Indices for Four Nested BRIEF Models.

\begin{tabular}{lrrrrrrrrr}
\hline Model & $\chi^{2}$ & $d f$ & $\chi_{\text {diff }}^{2}$ & $\chi^{2} / d f$ & CFI & SRMR & RMSEA & $\begin{array}{c}\text { Average off- } \\
\text { diagonal standardized } \\
\text { residual }\end{array}$ & $\begin{array}{c}\text { Largest } \\
\text { standardized } \\
\text { residual }\end{array}$ \\
\hline 1-factor (unity) & 470 & 27 & & 17.41 & .77 & .09 & .21 & .09 & .05 \\
2-factor & 172 & 26 & $298^{*}$ & 6.62 & .92 & .05 & .12 & .04 & .13 \\
3-factor & 130 & 24 & $42^{*}$ & 5.42 & .95 & .04 & .11 & .04 & .12 \\
4-factor & 124 & 21 & 6 & 5.91 & .95 & .04 & .12 & .12 \\
\hline
\end{tabular}

${ }^{*} p<.001$.

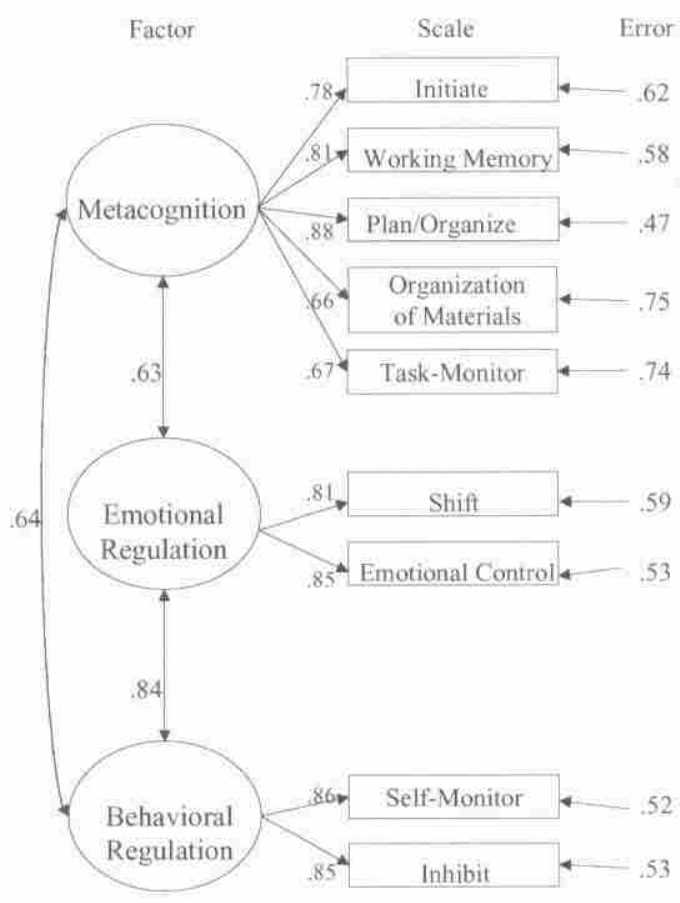

Fig. 1. Latent factor solution for Model 3. executive functions (ranging from $40 \%$ to $70 \%$ ), consistent with a fractionated, yet unified, view of executive functions (e.g., Miyake et al., 2000).

The model fit and, in particular, the elevated RMSEA (.11) and largest standardized residual values (.12) were further investigated via a review of the Lagrange Multiplier Test for the presence of correlated errors, which are not uncommon in the analysis of covariance structures (Byrne \& Shavelson, 1996). The specification of correlated errors between scales, however, must be derived from substantive theory, rather applied in a post hoc manner. In the case of the executive functions, a primary relation between inhibition and the other processes, including working memory, organization, and emotional control, has been posited (Barkley, 1997; Burgess, 1997). Examination of the Lagrange Multiplier Chi-square cumulative multivariate statistics indicated significant correlated errors between the BRIEF Inhibit scale and the Working Memory, Organization of Materials, and Emotional Control scales. As a result, the initial 3-factor model was respecified to 
estimate these three error covariances resulting in an improved model fit, where the CFI increased to .97 , the SRMR and RMSEA decreased to .03 and .08 respectively, and the $\chi^{2} / d f$ ratio was 3.4. Finally, the largest standardized residual reduced to 0.09 between Inhibit and Plan/ Organize,

\section{DISCUSSION}

The underlying factor structure of the BRIEF was examined to establish the best fit amongst four competing models for a sample of children with various clinical disorders. The present findings support a fractionated 3-factor model of executive function as the best fit for the clinical scales, with the Monitor scale subdivided into Self-Monitoring and Task-Monitoring scales. The model structure included Behavioral Regulation, Emotional Regulation, and Metacognition factors. The Behavioral Regulation factor was defined by the Inhibit and Self-Monitor scales, the Emotional Regulation factor was defined by the Emotional Control and Shift scales, and the Metacognition Factor was defined by the Initiate, Working Memory, Plan/Organize, Organization of Materials, and Task-Monitor scales. The 3-factor model had a significantly improved fit over the 1- and 2factor models while the 4-factor model offered no significant statistical improvements. Thus, parsimony would dictate the 3-factor model as the most appropriate structure for the 9-scale BRIEF.

The different factor structure of the BRIEF from past analyses deserves further explanation. Whereas the previous investigations with the 8 scale BRIEF using exploratory factor analysis yielded two factors, subdivision of the Monitor scale into two distinct components, Self-Monitor and Task-Monitor, allows for expansion of the BRIEF structure. In the resulting 3-factor model, the inhibitory behavior control dimension was differentiated from the emotional control dimension. That is, this model provided for two aspects of regulatory control - inhibition of external behavioral and internalized emotional control and flexibility. This model is more consistent with Barkley's (1997) view of executive function with: (1) inhibitory control having a unique and separable role; (2) emotional regulation playing an integral role, and; (3) metacognitive aspects of executive function forming a separable component (reconstitution). The metacognition factor retained the same scale definition in both the present 3 -factor model and the previously proposed 2-factor model (Gioia et al., 2000; Gioia, Isquith, Retzlaff, \& Pratt, 2001), remaining differentiated from behavioral and emotional regulatory dimensions. Of note are the high correlations among the three latent factors, reflecting the necessary interaction among these fractionated components of executive function.

The model identified in the present analyses, fractionating the supervisory system into three underlying processes, also is consistent in part with Shallice and Burgess (1991). Whereas the present methodology reflects less overall fractionation ( 3 factors vs. the 4 posited by Shallice \& Burgess, 1991), the model of a more unitary overarching general executive, was rejected. The results also lend partial support to Rabbitt's (1997) argument that common underlying processes may drive the behavioral manifestation of the executive functions rather than existing as independent encapsulated entities. The present model suggests that inhibitory behavioral regulation, emotional regulation, and metacognitive problem-solving are those underlying executive processes.

The present confirmatory factor analysis provides evidence for the validity of the BRIEF as a multi-dimensional measure of executive function based on its internal structure and consistency of the data-based model with theoretical models of executive function. The BRIEF's reliance on rating scale methodology also offers the advantage of ecological validity (Gioia \& Isquith, in press; Silver, 2000), capturing the integrated, multidimensional, relativistic nature of the executive system that often is demanded in real world situations (Goldberg \& Podell, 2000; Shallice \& Burgress, 1991). Although intending to clarify the nature of executive functions, the majority of experimental approaches rely on cognitive performance test methodologies, which may obscure the findings because of the confounded measurement characteristics of executive function tests. 
On the other hand, while the present behavioral rating methodology offers stronger ecological validity and generalizability, it also entails less experimental control and process specificity than that of laboratory tests.

An as yet untested methodology for modeling executive function that may balance the internal validity benefits of performance tests with the ecological validity benefits of behaviorallyanchored scales such as the BRIEF is to develop models incorporating both measurement approaches. Specifically, a multi-trait, multimethod approach can be used, where performance on traditional tests of discrete to complex functions administered in well controlled settings are combined with ratings of everyday behaviors on reliable measures of these same executive functions in the natural environment.

The investigation of competing factor models for the BRIEF with a sample of children with mixed clinical diagnoses has a distinct advantage in that it allows for greater generalization to clinical populations at large. In addition, the construct validity of the BRIEF merits further investigation in more specific populations. Indeed, executive function deficits, while characteristic of many disorders, may be expressed differently depending on the nature of the disorder (Gioia, Isquith, \& Kenworthy, in press; Ozonoff \& Jensen, 1999). These disorder-specific executive profiles may arise from disorder-specific underlying executive function structures. In order to understand how executive functions are impacted uniquely within specific clinical disorders, varying models of executive function should be contrasted within well-defined diagnostic groups. That is, both the behavioral expression of executive deficits, as well as specific models of executive function in specific diagnostic groups, should be explored.

While the present findings provide direct testing of alternative models of the BRIEF, several limitations should be acknowledged. First, the freeing of the correlated errors between the Inhibition scale and Working Memory, Emotional Control, and Organization of Materials scales, though based on substantive theory, was done on a post hoc basis. The measurement issues that are captured in the relations between these scales deserve further investigation. The possibly unique role of inhibition would be one area to examine. Even after these covariances were freed, the RMSEA remained elevated somewhat, indicating that the fit of the model could be improved further as this index reflects the presence of existing covariance that does not fit well within the defined 3-factor model. Further examination of the individual BRIEF scales suggests that the Organization of Materials and Task-Monitor scales have relatively lower relations $\left(R^{2}=.44\right.$, .45 , respectively) to the Metacognition factor than the Initiate, Working Memory, and Plan/Organize scales, and may share some responsibility for this elevated fit index. Interestingly, their fit was no better in the 4-factor model when constrained to load with each other, suggesting a different relation with the remaining executive function scales. These psychometric issues require further investigation, perhaps with validation on other normative and clinical samples.

A second limitation of this study is the inverse of its strength, that is, a focus on molar-level behavioral manifestations of executive function. The specificity of identifying particular discrete executive function processes may be reduced with this more global approach. As previously stated, a combined multi-trait, multi-method approach of modeling, with performance tests and the BRIEF, might provide a more complete view of the executive function landscape with specific cognitive processes and behavioral manifestations linked together.

Several future directions are suggested in terms of modeling executive function and the BRIEF. Examination of the model fit in specific relevant, clinical groups (e.g., Traumatic Brain Injury, ADHD, Autism Spectrum Disorders) would provide a useful window into the generality and specificity of model's executive function. In a related vein, examination of the BRIEF structure in the normative sample, possibly at several developmental points in time, also would contribute valuable information regarding the ontogenetic development of executive functioning. Multiple group comparisons might elucidate similarities and differences in the underlying structure of executive function in normally developing and clinical groups. Similar analyses with 
teacher ratings on the BRIEF would also be informative with respect to the generality and specificity of these models across settings and respondents.

Given the need for continued study of this 3factor model as discussed above, several implications can be drawn from the present study in terms of the clinical use of the BRIEF. It is reasonable to consider the clinical findings of the Parent Form of the BRIEF in terms of the child's regulatory function in three domains: cognitive problemsolving, control of behavior via inhibition, and flexible emotional control. These findings suggest that differing profiles of strength and weakness may emerge in terms of this 3-factor configuration. Further exploration of the clinical significance of varying profiles will further inform clinical interpretation.

With respect to current application of these findings to the clinical use of the published BRIEF Parent Form, clinicians have several options. One can interpret the standard published 8-scale inventory in terms of the 2factor Behavioral Regulation and Metacognition Indexes. Clinicians can also consider the findings of this study and examine the subdivision of the Monitor scale in terms of the Task-Monitor (Items 14, 21, 31, 60) and Self-Monitor (Items $34,42,52,63)$ components. In doing so, the items from the Task-Monitor subdivision would be interpreted together with the scales on the Metacognition Index, given its demonstrated relationship. Similarly, the items from Self Monitor subdivision would be interpreted together with the Inhibit scale in terms of inhibitory control. Finally, the Shift and Emotional Control scales would be interpreted in terms of the child's flexible emotional regulation. Such an analysis would provide a 3 -factor model of interpretation.

\section{ACKNOWLEDGEMENTS}

We are grateful to Lindy Jones for her editorial assistance in preparing the manuscript, and to Jacobus Donders for his helpful guidance in developing the paper. We also wish to acknowledge Laura Kenealy for her generosity in sharing data.

\section{REFERENCES}

Barkley, R.A. (1997). ADHD and the nature of selfcontrol. New York: Guilford Press.

Barkley, R.A. (2000). Genetics of childhood disorders: XVII. ADHD, Part 1: The executive functions and ADHD. Journal of American Academy of Child and Adolescent Psychiatry, 39. 1064-1068.

Bentler, P.M., \& Wu, E.J.C. (1995). EQS for Windows user's guide. Encino, CA: Multivariate Software.

Burgess, P. (1997). Theory and methodology in executive function research. In P. Rabbitt (Ed.), Merhodology of frontal executive function (pp. 81-116). East Sussex, UK: Psychology Press.

Byrne, B.M., \& Shavelson, R.J. (1996). On the structure of social self-concept for pre-, early and late adolescents: A test of the Shavelson, Hubner and Stanton (1976) model. Journal of Personality and Social Psychology, 70, 599-613.

Denckla, M.B. (1996). A theory and model of executive function: A Neuropsychological perspective. In G.R. Lyon \& N.A. Krasnegor (Eds.), Attention, Memory, and Executive Function (pp. 263-278). Baltimore, MD: Paul H. Brookes.

Gioia, G.A., \& Isquith, P.K. (2002). Two faces of Monitor: Thy self and thy task [Abstract]. Journal of the International Neuropsychological Society, 8 , 229.

Gioia, G.A., \& Isquith, P.K. (in press). Assessment of executive function in traumatic brain injury fram an ecological perspective, Manuscript submitted for publication.

Gioia, G.A., Isquith, P.K., Guy, S.C., \& Kenworthy, L. (2000). Behavior Rating of Executive Function. Lutz, FL: Psychological Assessment Resources.

Gioia, G.A., Isquith, PK., \& Kenworthy, L. (in press). Profiles of everyday executive function in acquired and developmental disorders. Child Neuropsychology.

Gioia, G.A., Isquith, P.K., Retzlaff, P.D., \& Pratt, B.M. (2001). Modeling executive functions with everyday behaviors: A unitary or fractionated system? Brain and Cognition, 47, 203-207.

Goldberg, E., \& Podell, K. (2000). Adaptive decision making, ecological validity, and the frontal lobes. Journal of Clinical and Experimental Neuropsychology, 22, 56-68.

Goldman-Rakic, P. (1987). Circuitry of primate prefrontal cortex and regulation of behavior by representational memory. In V. Mountcastle, F. Plum, \& S.R. Geiger (Eds.), Handbook of physiology: The nervous system (Vol. 5, Part 1, pp. 373417). NY: Oxford University Press. 
Hu, L., \& Bentler, P.M. (1998). Fit indices in covariance structure modeling: Sensitivity to underparameterized model misspecification. Psychological Methods, 3, $424-453$.

Hu, L., \& Bentler, P.M. (1999), Cutoff criteria for fit indices in covariance structure analysis: Conventional criteria versus new alternatives. Structural Equation Modeling, 6, 1-55.

Kelly, T.P. (2000). The development of executive function in school-aged children. Clinical Neuropsychological Assessment, 1, 38-55.

Levin, H.S., Culhane, K.A., Hartmann, J., Evankovich, K., Mattson, A.J., Harward et al. (1991). Developmental changes in performance on tests of purported frontal lobe functioning. Developmental Neuropsychology, 7, 377-395.

Miyake, A., Friedman, N.P., Emerson, M.J., Witzki, A.H., Howerter, A., \& Wagner, T.D. (2000). The unity and diversity of executive functions and their contributions to complex "frontal lobe" tasks: A latent variable analysis. Cognitive Psychology, 41, 49-100.

Ozonoff, S., \& Jensen, J. (1999). Brief report: Specific executive function profiles in three neurodevelopmental disorders. Journal of Autism and Developmental Disorders, 29, 171-177.
Pennington, B.F., \& Ozonoff, S. (1996). Executive functions and developmental psychopathology. Journal of Child Psychology and Psychiatry and Allied Disciplines, 37, 51-87.

Rabbitt, P. (1997). Introduction: Methodologies and models in the study of executive function. In P. Rabbitt (Ed.), Methodology of frontal executive function (pp. 1-38). East Sussex, UK: Psychology Press.

Shallice, T., \& Burgess, P. (1991). Higher-order cognitive impairments and frontal lobe lesions in man. In H. Levin, H. Eisenberg, \& A. Benton (Eds.), Frontal lobe function and dysfunction (pp. 125138). New York: Oxford University Press.

Silver, C.H. (2000). Ecological validity of neuropsychological assessment in childhood traumatic brain injury. Journal of Head Trauma Rehabilitation, 15, 973-988.

Welsh, M.C., \& Pennington, B.F. (1988). Assessing frontal lobe functioning in children: Views from developmental psychology. Developmental Neuropsychology, 4, 199-230.

Welsh, M.C., Pennington, B.F., \& Grossier, D.B. (1991). A normative-developmental study of executive function: A window on prefrontal function in children. Developmental Neuropsychology, 7, 131-149. 
Copyright $@ 2003$ EBSCO Publishing 